\title{
Mobile 3D Mapping with A Low-Cost UAV-Based LIDAR System
}

\author{
Phan, A. T. T., ${ }^{1,2^{*}}$ Dang, C. B. V. ${ }^{1,2}$ and Takahashi, K. ${ }^{3}$ \\ ${ }^{1}$ Department of Geomatics Engineering, Faculty of Civil Engineering, Ho Chi Minh University of \\ Technology, 268 Ly Thuong Kiet Street, Ward 14, District 10, Ho Chi Minh City, Vietnam \\ E-mails: ptathu@hcmut.edu.vn,dvcbang@hcmut.edu.vn \\ ${ }^{2}$ Vietnam National University Ho Chi Minh City, Linh Trung Ward, Thu Duc District, Ho Chi Minh City, \\ Vietnam \\ ${ }^{3}$ Nagaoka University of Technology, 1603-1, Kami-Tomioka, Nagaoka, Niigata 940-2188, Japan \\ E-mails: ktakaha@nagaokaut.ac.jp \\ *Correspondence Auhtor \\ DOI: https://doi.org/10.52939/ijg.v17i3.1905
}

\begin{abstract}
Recently, many UAVs (unmanned aerial vehicles) based on LiDAR (light detection and ranging) systems have been developed for various purpose because of the effective of LIDAR technique and low-cost UAV. In this study, the accuracy of point clouds generated by the developed for a low-cost UAV-based LiDAR systems is evaluated. The system consisting of a multi-beam laser scanner-Velodyne VLP 16 and DJI M600 UAV. The experimental site is undulation with less object in Nagaoka city, Niigata Prefecture, Japan Twelve reflectance makers are arranged as ground control point for the positioning evaluating process. The observed data was collected on Nov. 8th, 2019 with three different flight height at 10m, $20 \mathrm{~m}$ and 30m. For generating the point clouds, the mounting parameters and sensor parameters are combined. The generated point clouds are corrected by applying bias correction and the 7 parameters transformation. The result is validated using three different experimental setups with three various flight height which indicate that the most accurate and reliable results are obtained. As a result, the point clouds after calibrating attained an accuracy of approximate $0.2 \mathrm{~m}$ in vertical and horizontal for both correction methods. In conclusion, the point cloud accuracy is not good enough for generating the topographic map at large scale. However, the stable results and the present accuracy are good for other purposes with less accuracy requirement such as monitoring the crop growth.
\end{abstract}

\section{Introduction}

Recently, the small, low cost, UAV (Unmanned aerial vehicle) platforms have been rapidly developed. As a result, they become the costeffective platform solutions for mapping purposes or collecting geospatial data. Untill now, there are many types of UAVs and sensors, and systems dedicated to topographic mapping. Typically, camera systems are usually mounted UAV platforms but other sensors, such as LiDAR (Light Detection and Ranging), HSI (HyperSpectral Imagery) are increasingly payed attention (Niethammer et al., 2012, Adão et al., 2017 and Angel et al., 2019).

Nowadays, LiDAR sensors are utilized as an effective tool for collecting accuracy 3D geospatial data in short time. Number of LIDAR types, recently, increase rapidly; therefore, LiDAR sensors can enhance performance which small size and cost effective. Moreover, the GNSS/INS direct georeferencing technology is improved. As a result, LiDAR sensor -based data acquisition is getting

popular and known as a cost-effective technique. LiDAR technique can be applied for civilian and defense applications, to archaeological, and environmental studies such as generating high accuracy digital surface model, digital elevation model with high resolution (Mcintosh et al., 2000 and Ma et al., 2005) digital building model generation, transportation monitoring, telecommunications.

Many mobile LIDAR systems have been developed for various purposes. They can be manned or unmanned system. UAV can be used as an effective platform for developing the system. A UAV- based laser scanner system is considered as a useful tool to quickly collect 3D point cloud data of the crop or monitoring the forest such as estimating canopy height (Ehlert et al., 2010, Zawawi et al., 2015 and Sibona et al., 2017), canopy structure (Rice et al., 2005), carbon stork (Maan et al., 2015) and vertical plant density profile (Hosoi and Osama 2006, 2009). Most of low-cost UAV limits the 
weight of payload. Therefore, the chosen LiDAR sensors for developing UAV-based system need to be commensurate with the payload limitation as well as their effectiveness for expected application.

For developing mobile mapping systems and robotics applications, the Velodyne laser scanners have been used because of their cost-effective and highly scan speed. For this, many researches have been carried out to model the systematic errors, calibrating the LiDAR systems as well as correcting the output 3D point clouds (Glennie et al., 2016 and Ravi et al., 2018). For collecting geospatial data, georeferencing is an important issue. Therefore, the outdoor calibration of mobile LIDAR system has been studied for improve the mapping accuracy of the generated point cloud. In this study, we try to generate and test the accuracy of 3D point cloud generated from our developed UAV- based LiDAR system using Velodyne VLP 16 laser scanner. Firstly, all components involved in the UAV- based LiDAR system used in this research is introduced. Then, the mathematical model for generating 3D point cloud is shown. For this step, the bias offset between origins of different reference frames, the rotation angle of the Gimbal and UAV and the grid convergence are considered carefully. Finally, we focus on evaluating and calibrating the accuracy of generated point cloud.

\section{System Description}

For ensuring the requirement of reducing equipment cost, and convenience and ease of carrying all selected devices are low-cost, compact, and lightweight. In detail, for developing the UAV-based LiDAR system, the DJI M600 UAV platform is used (Figure 1a). This platform is designed for professional aerial mapping applications, including the A3 flight controller, Lightbridge 2 transmission

(b) (a)

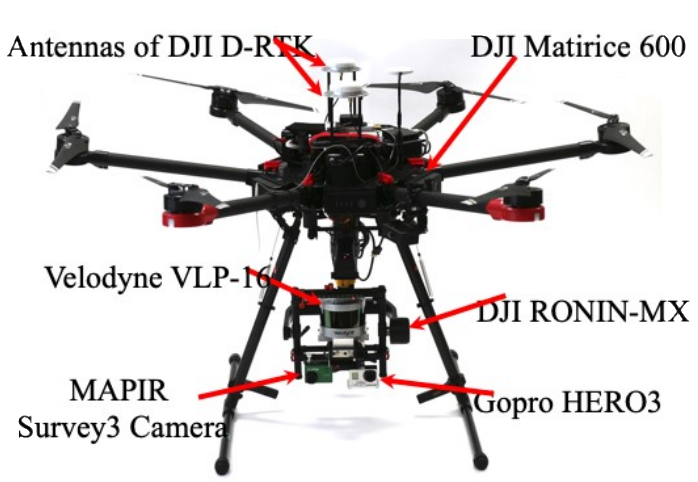

system, Intelligent Batteries and Battery Management system (Velodyne LiDAR, 2018). Its total weight with batteries is greater than $9.0 \mathrm{~kg}$. Maximum take-off weight is $15.5 \mathrm{~kg}$ and maximum payload is $6.0 \mathrm{~kg}$. Its max flight altitude above sea level is $2500 \mathrm{~m}$ and max speed at $18 \mathrm{~m} / \mathrm{s}$ with nowind environment. On flying at $10 \mathrm{~m}$ above sea level in a no-wind environment, this UAV can flight 16 minutes with maximum payload. The selected LiDAR sensor is Velodyne VLP-16 Puck (Figure 1b). It is a small LiDAR unit with a $360^{\circ}$ horizontal field of view and a $30^{\circ}$ vertical field of view, with $15^{\circ}$ up and down. Its maximum detection range is $100 \mathrm{~m}$. This sensor uses an array of 16 infrared (IR) lasers paired $(903 \mathrm{~nm})$ with IR detectors to measure distances to target objects. All components are assembled within a compact, weather-resistant housing. Each laser/detector pair can fire each laser approximately 18,000 times per second, providing a set of $3 \mathrm{D}$ point data in real-time. As a result, VLP16 sensor having 16 laser/detector pairs enables measurements of up to 300,000 data points per second or double that in dual return mode. This device is mounted on a DJI Ronin-MX gimbal to maintain its horizontal attitude. Moreover, the Camera Gopro Hero 3 and Survey3 Cameras MAPIR CAMERA are also installed to record the video during data collecting experiment (Figure 1a). However, these video data are not be analysized in this study.

\section{Data Acquisition}

For convenience performing the experiment, an experiment site is selected beside Nagaoka University of technology in Niigata Prefecture, Japan. Before performing the experiment, twelve square markers are arranged on the experimental site (Figure 2).

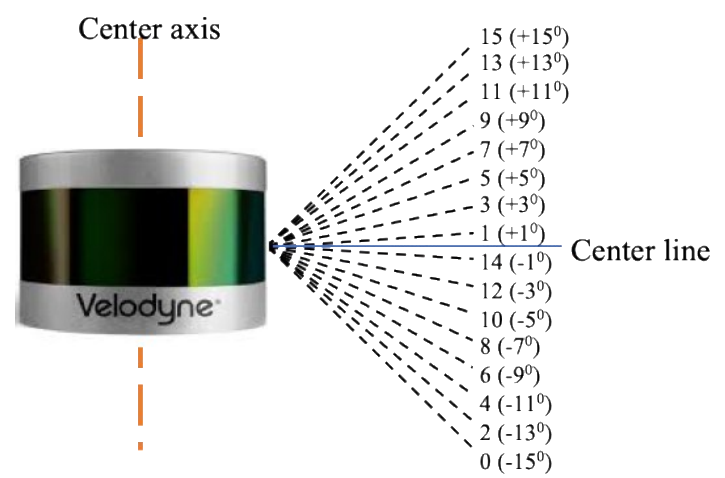

Figure 1: The UAV-based LiDAR system. (a) All components of the system and the laser ID and vertical angle of Velodyne VLP-16 


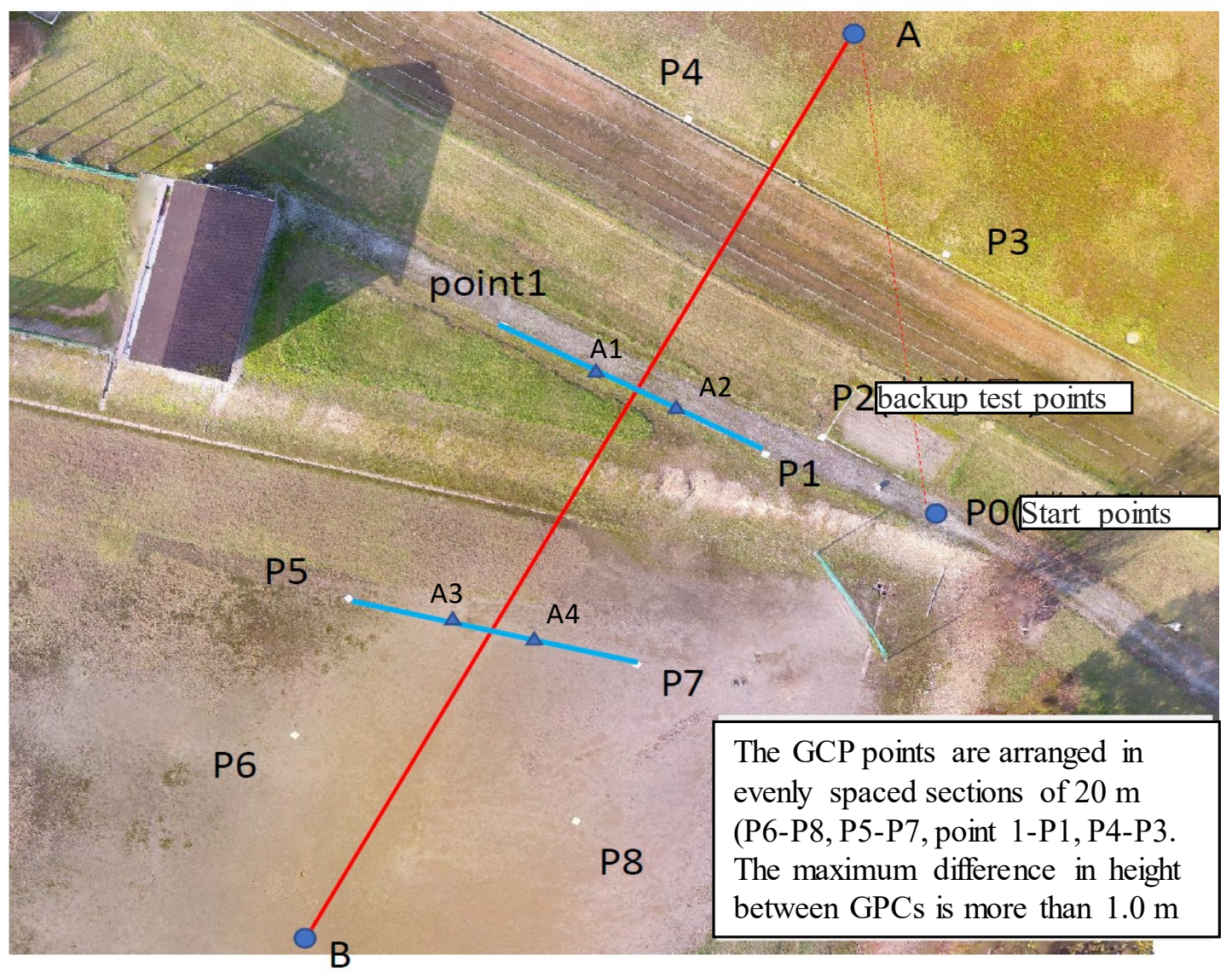

Figure 2: The experimental site in Nagaoka city, Niigata Prefecture, Japan. There are twelve reflectance markers arranged on the site. Line $\mathrm{AB}$ shows the flight direction

Table 1: The mapping coordinates of GCPs

\begin{tabular}{|c|c|r|c|}
\hline ID & $\mathbf{X}(\mathbf{m})$ & $\mathbf{Y ~ ( m )}$ & $\mathbf{Z}(\mathbf{m})$ \\
\hline A1 & 157712.583 & 24406.232 & 53.668 \\
\hline A2 & 157707.360 & 24416.586 & 53.648 \\
\hline A3 & 157693.050 & 24396.753 & 52.461 \\
\hline A4 & 157690.479 & 24408.062 & 52.448 \\
\hline P1 & 157705.475 & 24420.330 & 53.675 \\
\hline P3 & 157720.756 & 24432.011 & 53.595 \\
\hline P4 & 157730.239 & 24414.400 & 53.584 \\
\hline P5 & 157693.980 & 24392.666 & 52.468 \\
\hline P6 & 157683.709 & 24389.298 & 52.500 \\
\hline P7 & 157689.564 & 24412.167 & 52.446 \\
\hline P8 & 157677.801 & 24408.413 & 52.469 \\
\hline
\end{tabular}

They are considered as ground control points (GCPs) for evaluating the accuracy of 3D point cloud. Their coordinates observed by a RTK technique and directly measurement using a total station observation (Table 1). For general evaluating the generated 3D point clouds, the chosen experiment site must not flat. The surface elevation changes over the fields. The maximum difference in height between 12 GCPs is greater than $1.0 \mathrm{~m}$. Moreover, there is a small house inside the target 
field for testing the point cloud visualization. The experiment was carried out on Nov. 8th, 2019 with three different flight height at $10 \mathrm{~m}, 20 \mathrm{~m}$ and $30 \mathrm{~m}$. For collecting data, a base station for a DJI -DRTK on a M600 was set up. The system was moved to the start point and the VLP 16 was controlled by a personal computer. The VLP16 was turned on and started to record data using VeloView. When the experiment completed, DJI assistant2 software was used to download the flight log file from the flight controller (DJI-A3). Then, this flight log file was uploaded to the Web site (PhantomHelp) to generate flight log data using "DJI Flight Log Viewer. In total, there were 3 flight log files and 3 PCAP files recorded for this experiment. In this current system the VLP16 and a flight controller are not connected. Therefore, they have a different GPS receiver to record GPS time, and the recording start time of VLP16 was always earlier than flight log's time. As a result, the GPS time in two data sources must be synchronized for the $3 \mathrm{D}$ point cloud generating process.

\section{Methodology and Results}

\subsection{Mathematical Model for a UAV-Based LiDAR $3 D$ Point Cloud Generation}

The developed UAV-based LiDAR system in this study consisting of a multi-beam laser scanner involves five reference frames-LiDAR sensor frame, GIMBAL frame, IMU frame, GNSS frame and local mapping frame. For generating the 3D point cloud, the foremost step is to establish the mathematical relationship between these mentioned reference frames. For defining the LiDAR sensor frame, its origin is defined at the laser beams firing point where is $37.7 \mathrm{~mm}$ above the sensor base, on the center axis. The $\mathrm{z}$-axis is defined as the center axis of the LiDAR sensor (VLP 16). For each beam, the sensor reports distances relative to itself in spherical coordinates (radius $r$, vertical angle $\omega$, azimuth $\alpha$ ) as shown in Figure 3. Therefore, $\mathrm{Y}$ axis point to direction of 0 degree of azimuth. The $\mathrm{X}$ axis is defined from right-hand rule. So, the $3 \mathrm{D}$ coordinates of a point relative to the LiDAR sensor coordinate system is expressed by Equation (1).

$$
P_{L S}=\left(\begin{array}{c}
x_{L S} \\
y_{L S} \\
z_{L S}
\end{array}\right)=\left(\begin{array}{c}
-r \cdot \cos \omega \cdot \sin \alpha \\
r \cdot \cos \omega \cdot \sin \alpha \\
r \cdot \sin \omega
\end{array}\right)
$$

Eqaution 1

Where:

$\left(X_{L S}, Y_{L S}, Z_{L S}\right)^{T}$ : The coordinate of scanning point in LiDAR sensor coordinate system.

$r$ : The observed range

$\omega$ : The vertical angle

$\alpha$ : The azimuth angle

The LiDAR sensor is mounted on a Ronin-MX gimbal assembled to the UAV. The Gimbal frame is aligned with IMU frame. The LiDAR sensor is mounted on a Ronin-MX gimbal assembled to the UAV for maintaining the LiDAR sensor attitude. The Gimbal frame is set, aligning with IMU frame. The IMU is assembled on the UAV with its Y-axes aligned along flight line directions. It is assumed that the $\mathrm{Z}$-axis of IMU is perfectly aligned with the vertical direction of the local mapping frame. The $\mathrm{X}$-axis of IMU is identified by right-hand rule and it aligned along the starboard of the UAV (Figure 4). The GNSS receiver provides the position of UAV in time. From the flight log file, the position of GNSS antenna is expressed as the latitude and longitude. Those mention coordinates are converted to 3D coordinates in the local coordinated system. Three rotation angles (yaw, pitch and roll) achieved from inertial measurements unit (IMU) show the relationship between the local mapping frame and IMU body frame. In addition, the flight controller of used UAV is a DJI A3 with a DJI D-RTK. It has a Dual GNSS antenna to output UAV heading angle (yaw angle). (a)

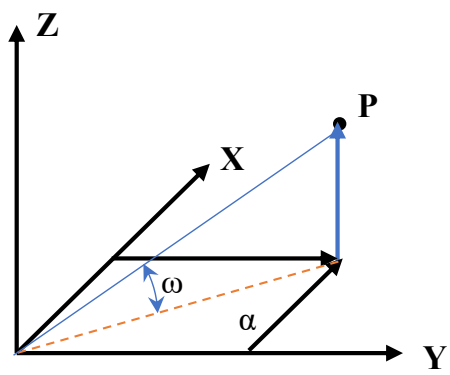

(b)

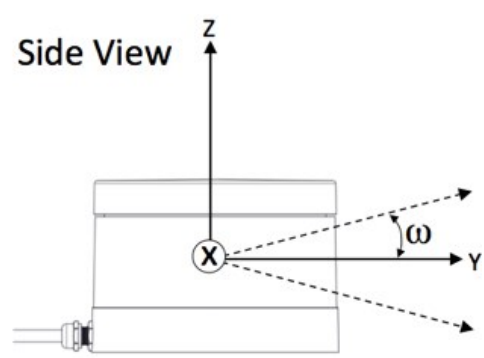

(c)

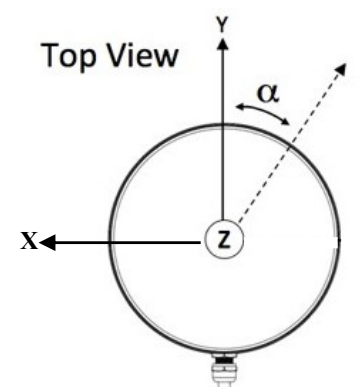

Figure 3: The position of data point in LiDAR sensor coordinate system. (a) The spherical coordinates, (b) vertical angle $\omega$ and (c) azimuth angle $\alpha$ 
(a)

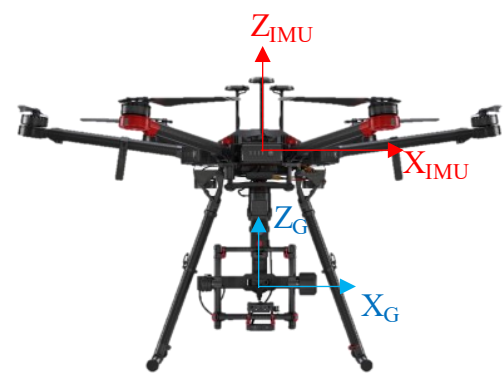

(b)

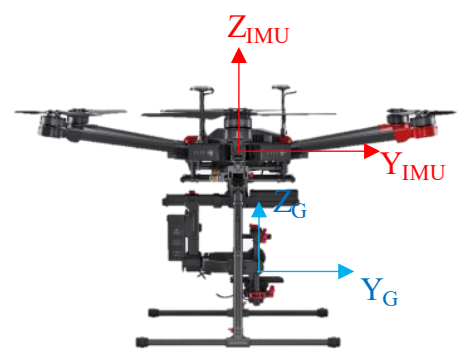

(c)

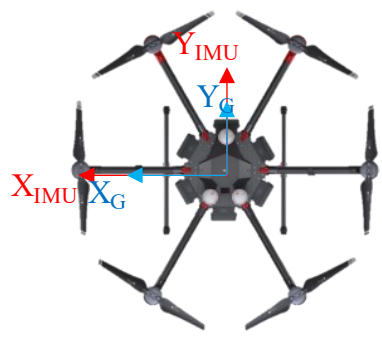

Figure 4: Illustration of IMU body frame and Gimbal frame in (a) front view, (b) slide view and (c) top view

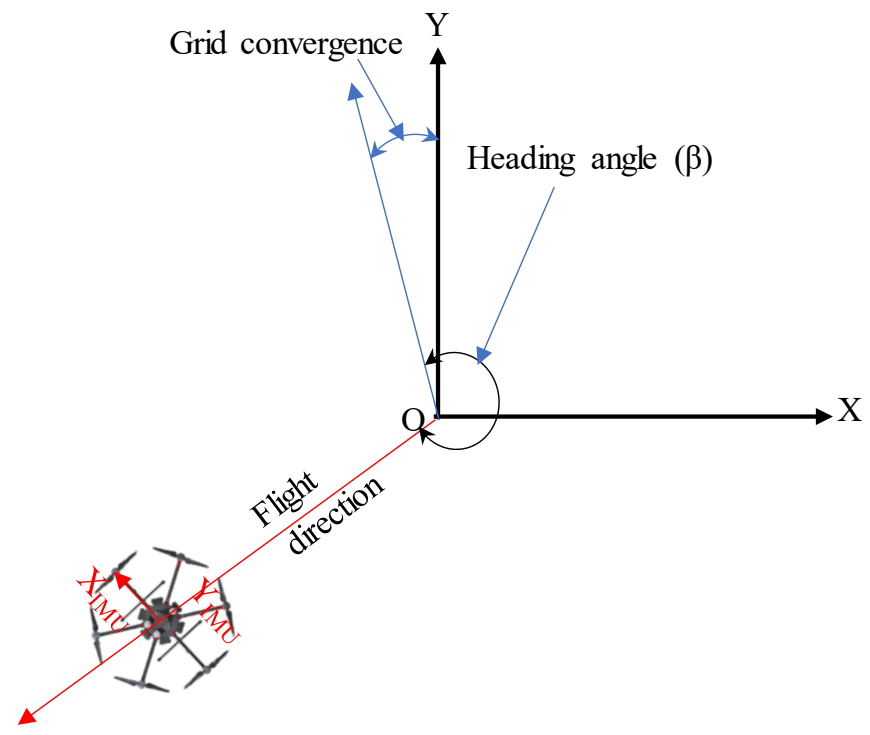

Figure 5: Illustration of relationship between heading angle and grid convergence

It shows the angle from the true north and the magnetic declination is ignored. For generating the 3D point clouds, the grid convergence should be adjusted to the heading angle (Figure 5). Moreover Ravi et al., (2018) shows the impact of bias between other reference frame origins on the generated 3D point cloud quality. Therefore, specifies offset vectors between the IMU frame and other reference frames are also measured and adjusted to the result (Figure 6). As a result, a point $\mathrm{P}$ acquired from the system can be identified in the local mapping coordinate system using equation (2).

$$
\boldsymbol{P}=\left(\left(\boldsymbol{R}_{G} \boldsymbol{P}_{L S}+\boldsymbol{B}_{G}^{L S}\right) \boldsymbol{R}_{L S}+\boldsymbol{B}_{I M U}^{G}\right) \boldsymbol{R}_{I M U}+\boldsymbol{B}_{G N S S}^{I M U}+\boldsymbol{P}_{U A V}
$$

Equation 2

Where:

$\boldsymbol{P}=(X, Y, Z)^{T}$ is the coordinate of scanning point in the $8^{\text {th }}$ of the Japan orthogonal coordinate system (the local coordinate system).

$\boldsymbol{R}_{G}$ : The rotation matrix of Gimbal, three rotation angles come from flight log file.
$\boldsymbol{P}_{L S}=\left(X_{L S}, \quad Y_{L S}, \quad Z_{L S}\right)^{T}:$ The coordinate of scanning point in LiDAR sensor coordinate system.

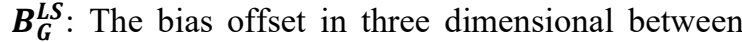
LiDAR frame and Gimbal frame origin. $\boldsymbol{R}_{L S}$ : The rotation matrix of the LiDAR sensor.

$\boldsymbol{B}_{I M U}^{G}$ : The bias offset in three dimensional between Gimbal frame and IMU frame origin.

$\boldsymbol{R}_{I M U}$ : The rotation matrix of the UAV, pitch and roll rotation angles come from flight log file, the yaw angle is computed from heading angle and the grid convergence.

$\boldsymbol{B}_{G N S S}^{I M U}$ : The bias offset in three dimensional between GNSS receiver frame and IMU frame origin.

$\boldsymbol{P}_{U A V}=\left(X_{U A V}, \quad Y_{U A V}, Z_{U A V}\right)^{T}$ : the position of UAV in local coordinate system is calculated from the comes from the latitude and longitude in flight $\log$ file.

\subsection{Generating 3D Point Cloud Generation}

According to equation 2 , all mounting parameters and sensor parameters must be identified for the 3D point cloud generating process. First of all, the 
PCAP file is visualized in Veloview application and data for each scanning frame is converted to a CVS file. From this file, the coordinate system of points in LiDAR framed is calculated from observed range, vertical and azimuth angle (equation (1)). As mentioned above, the observed data and flight log data are synchronized by GPS time. For collecting data, the Gimbal is adjusted an angle of approximate 90 degrees to make sure that the VLP 16 face to the ground surface. Three rotation angles of the gimbal will be obtained from the flight log file. However, the center line of VLP 16 is not nadir and, the measured the oblique angle is 9 degrees forward to nadir direction. Then, all bias offset values mentioned in equation 2 are measured (Figure 5). The position of GNSS antenna and IMU rotation angle are also obtained from flight log file. From those mentioned data, a script is developed from $\mathrm{R}$ language to generate the $3 \mathrm{D}$ point cloud using equation 2 . As a result, the $3 \mathrm{D}$ point cloud generated from experimental data is displayed in Figure 7. The difference in flight height lead to the difference of cover area and scanning point density. In general, all point clouds clearly show the image of 12 GCP and the small house in experiment site. However, the square shape of reflectance makers is only clearly visualized on $3 \mathrm{D}$ point cloud collected at $10 \mathrm{~m}$ of flight height but other flight heights.

\subsection{Georeferencing}

The coordinates of 12 markers (GCPs) are used for testing the accuracy of generated 3D point cloud. First of all, the coordinates of 12 GCPs are measured directly from the point cloud using cloud compare application. They are directly compared to the original coordinates of GCPs measured by the total station. It is clear that there is the bias between LiDAR-derived height and original height of 12 GCPs (Table 2). The horizontal RMSE in three flight height is several meters. This is the result of low accuracy of GNSS positioning system. However, the vertical RMSEs in three cases are greater than $20 \mathrm{~m}$. The difference between them is explained by the geoid undulation between the local preference ellipsoid and the geoid along with low accuracy of GNSS positioning system. In a small area of this study, the geoid and ellipsoid surface are assumed as two parallel planes. Moreover, the image of 12 reflectance markers appear in the EastNorth of their really location. (a)

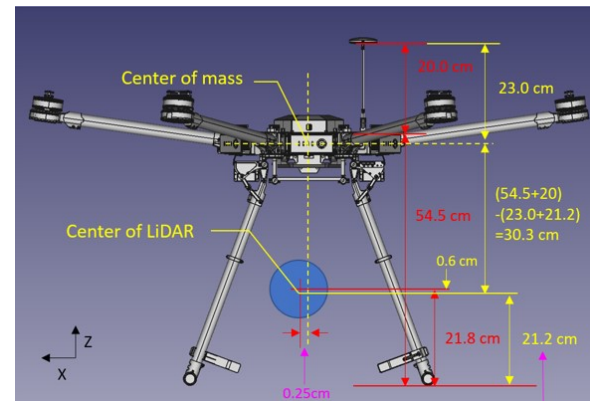

(b)

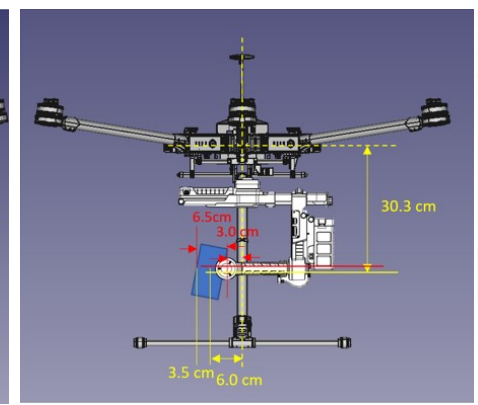

(c)

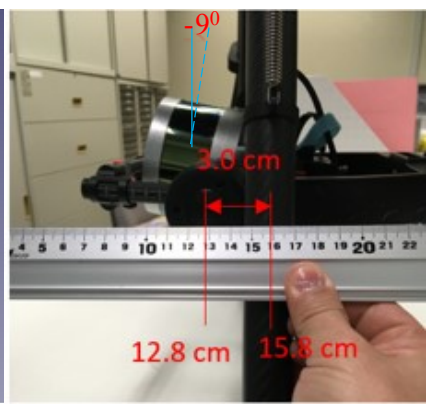

Figure 6: Measured offset values between (a) LiDAR sensor frame and IMU-body frame, (b) LiDAR sensor frame and Gimbal frame, and (c) the inclination of the LiDAR sensor

(a)

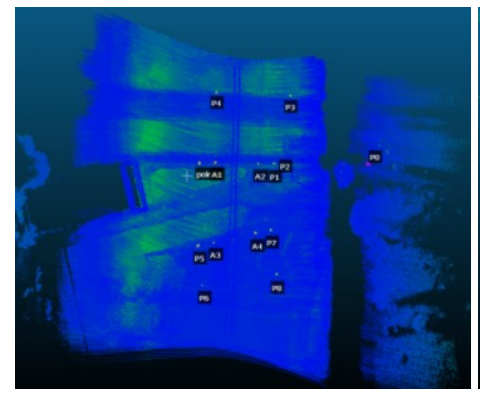

(b)

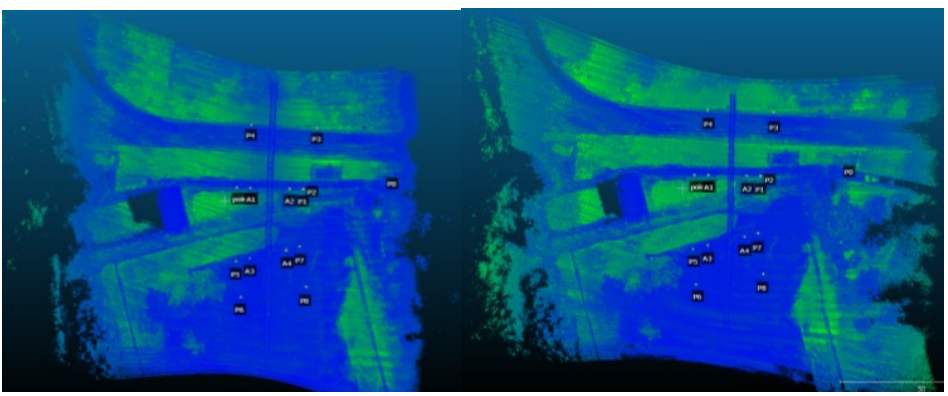

Figure 7: Visualization of 3D point cloud collected on Nov, $8^{\text {th }}, 2019$ at (a) $10 \mathrm{~m}$, (b) $20 \mathrm{~m}$ and (c) $30 \mathrm{~m}$ of flight height. The difference in flight height lead to the difference of cover area and scanning point density 
Table 2: The errors of 12 GCPs before applying any correction method

\begin{tabular}{|c|c|c|c|c|c|c|c|c|c|}
\hline \multirow{3}{*}{ ID } & \multicolumn{9}{|c|}{ Absolute errors } \\
\hline & \multicolumn{3}{|c|}{$10 \mathrm{~m}$ flight height } & \multicolumn{3}{|c|}{$20 \mathrm{~m}$ flight height } & \multicolumn{3}{|c|}{$30 \mathrm{~m}$ flight height } \\
\hline & $\mathbf{E x}(\mathbf{m})$ & Ey (m) & $\mathbf{E z}(\mathbf{m})$ & $\operatorname{Ex}(\mathbf{m})$ & Ey (m) & $\mathbf{E z}(\mathbf{m})$ & Ex (m) & Ey (m) & $\mathbf{E z}(\mathbf{m})$ \\
\hline A1 & 3.846 & 3.284 & 26.440 & 3.995 & 3.504 & 21.529 & 4.520 & 3.674 & 21.621 \\
\hline $\mathrm{A} 2$ & 4.100 & 3.386 & 26.542 & 4.051 & 3.541 & 21.731 & 4.756 & 3.813 & 21.655 \\
\hline A3 & 4.062 & 3.292 & 26.546 & 4.508 & 3.702 & 21.618 & 4.445 & 3.837 & 21.755 \\
\hline A4 & 4.285 & 3.443 & 26.492 & 4.581 & 3.680 & 21.619 & 4.695 & 4.052 & 21.608 \\
\hline $\mathrm{P} 1$ & 4.151 & 3.569 & 26.550 & 4.069 & 3.612 & 21.778 & 4.841 & 3.946 & 21.757 \\
\hline P3 & 3.797 & 3.594 & 26.589 & 3.739 & 3.683 & 21.791 & 5.394 & 3.995 & 21.847 \\
\hline P4 & 3.347 & 3.295 & 26.319 & 3.667 & 3.587 & 21.502 & 4.967 & 3.678 & 21.744 \\
\hline P5 & 4.000 & 3.244 & 26.554 & 4.319 & 3.819 & 21.508 & 4.409 & 3.817 & 21.662 \\
\hline P6 & 4.176 & 3.178 & 26.442 & 4.283 & 4.004 & 21.309 & 4.569 & 3.820 & 21.598 \\
\hline P7 & 4.259 & 3.556 & 26.511 & 4.606 & 3.697 & 21.624 & 4.847 & 3.883 & 21.986 \\
\hline P8 & 4.474 & 3.564 & 26.644 & 4.843 & 4.001 & 21.797 & 4.865 & 4.161 & 21.960 \\
\hline point1 & 3.819 & 3.174 & 26.447 & 4.037 & 3.432 & 21.454 & 4.301 & 3.672 & 21.294 \\
\hline
\end{tabular}

Table 3: Absolute distance errors

\begin{tabular}{|l|l|l|l|l|l|l|}
\hline \multirow{2}{*}{ Flight height } & \multicolumn{6}{|c|}{ Distance between Two Points (m) } \\
\cline { 2 - 7 } & P3-P4 & P4-P8 & P8-P6 & P6-P3 & P4-P6 & P3-P8 \\
\hline $10 \mathrm{~m}$ & -0.78 & -4.75 & 2.72 & -5.13 & -5.18 & -5.63 \\
\hline $20 \mathrm{~m}$ & -0.78 & -5.14 & 3.13 & -5.83 & -5.65 & -6.16 \\
\hline $30 \mathrm{~m}$ & 0.07 & -5.17 & 3.29 & -5.87 & -5.82 & -5.17 \\
\hline
\end{tabular}

Table 4: The calculated 7 parameters

\begin{tabular}{|l|l|l|l|l|l|l|l|}
\hline \multirow{2}{*}{$\begin{array}{l}\text { Flight } \\
\text { height }\end{array}$} & \multicolumn{4}{|l|}{ Translation (m) } & \multicolumn{2}{l|}{ Scale } & \multicolumn{3}{l|}{ Rotation angle (minutes) } \\
\cline { 2 - 8 } & $\mathbf{T x}$ & Ty & Tz & s & rx & ry & rz \\
\hline $10 \mathrm{~m}$ & -1599.354 & 414.402 & -92.231 & 1.009 & 12.7 & 0.0 & -14.1 \\
\hline $20 \mathrm{~m}$ & -2567.116 & -494.445 & 122.099 & 1.016 & -17.5 & 0.0 & 2 \\
\hline $30 \mathrm{~m}$ & 1286.635 & -1254.260 & 6.266 & 0.993 & -1.6 & 0.0 & 30.9 \\
\hline
\end{tabular}

In the simplest case, the position of reflectance markers is simply shifted by the mean bias distance. Therefore, the simple bias correction method is applied. For evaluating the corrected result, four GCPs P3, P4, P6 and P8 closed to four corners are chosen for the correction process. The rest of GCPs become check points. By taking the average of these difference in coordinates of 4 chosen GCPs and adjusting to the point coordinates, the absolute errors in horizontal and vertical of check points decreased to several decimeters in three cases of flight height. In detail, the vertical RMSE and horizontal errors in case of $10 \mathrm{~m}, 20 \mathrm{~m}$ and $30 \mathrm{~m}$ flight height are $0.094 \mathrm{~m}, 0.148 \mathrm{~m}$ and $0.193 \mathrm{~m}$ and horizontal RMSEs are $0.221 \mathrm{~m}, 0.268 \mathrm{~m}$ and 0.282 $\mathrm{m}$, respectively. According to the result, the errors increase along with the increase of flight height.

It is expected that the horizontal positioning accuracy of point cloud could be increased. For checking the type of geometric distortion, the distances between four chosen GCPs is computed form their coordinated and compared to their LiDAR-derived distances (Table 3). According to the results displayed in Table 3, for each distance, its errors in three cases are similar. However, the distance errors of difference lines differ to each 
other. Therefore, the geometric distortion might include rotating, scaling, shearing and translating. For this situation, 7 parameters transformation is expected to fix the problems with 4 GCPs (P3, P4, P6 and P8). Other GCPs will become checked points to evaluate the absolute accuracy of point cloud after applying the correction method. All 7 parameters are computed by applying least square method (Table 4). According to the results, the scaling factor in three cases are almost equal 1.0, and all rotation angle area less than 30 minutes. As a result, the absolute errors in horizontal and vertical of 8 check points are several decimeters in three cases of flight height. In detail, the vertical RMSE and horizontal errors in case of $10 \mathrm{~m}, 20 \mathrm{~m}$ and $30 \mathrm{~m}$ flight height are $0.164 \mathrm{~m}, 0.170 \mathrm{~m}$ and $0.234 \mathrm{~m}$ and horizontal RMSEs are $0.199 \mathrm{~m}, 0.212 \mathrm{~m}$ and 0.263 $\mathrm{m}$, respectively. These results show that, bias and 7 parameters transformation method give the same accuracy of the generated point cloud in this study.

\section{Discussion}

This study is carried out to evaluate the accuracy of the 3D point cloud generated from observed data collected by a UAV- based LiDAR system. For developing the UAV-based LiDAR system, the multi beam laser scanner Velodyne VLP 16 is mounted on the DJI M600 UAV. This system flighted over the experimental site at three difference flight height of $10 \mathrm{~m}, 20 \mathrm{~m}$ and $30 \mathrm{~m}$ to collect the image of objected including 12 GCPs arranged over the undulating surface.

For generating the $3 \mathrm{D}$ point cloud, the relationship between five reference frames is figured out and expressed as equation 2. All scripts are coded on $\mathrm{R}$ language to generating the point clouds. As a result, the image target area is visualized from three generated point clouds. At high flight height of $30 \mathrm{~m}$ the image of object may not clear. At the flight height of $10 \mathrm{~m}$, the image of GPSs is clear in shape. However, the objects hidden behind the house cannot be observed because of low flight height.

For simple the data analysis process in the small area, the accuracy of point in vertical and horizontal can be separately checked by directly compare the difference of coordinate of 12 GCPs in two data sets (Lidar and original data set). The GNSS signal returns the ellipsoidal height whereas the height of point in the local coordinate system using the geoid surface as reference surface. Therefore, the large absolute error in vertical direction is cause by the undulation of geoid surface and the difference between geoid and local preference ellipsoid. The difference of height bias computed from average height errors of 12 GCPs in three different flight height is caused by the vertical positioning accuracy of the system. The GPS data supported by DJI -DRTK of single frequency is not absolutely correct. By applying the bias correction method with 4 GCPs, the positiong accuracy is increased. In detail, the root mean square errors in vertical direction $\left(\mathrm{RMSE}_{\mathrm{Z}}\right)$ in three flight height is approximated 0.2 $\mathrm{m}$ whereas the is approximated $0.3 \mathrm{~m}$. By checking the distance errors, the geometric distortion might include rotating, scaling and shearing and offset distorsion. The 7 parameters transformation has been applied with 4 GCPs near to four corners of the experimental site. As a results, the achieved RMSE is similar to the results of applying bias correction method. Therefore, in small area with maximum difference in height is more than $1.0 \mathrm{~m}$, the bias correction method can be applied to achieve the positioning accuracy of less than $0.3 \mathrm{~m}$.

\section{Conclusion}

In this paper, we identified the mathematical model of generating the 3D point cloud from observed data collected by the developed for UAV-based LiDAR systems consisting of a spinning multi-beam laser scanner. This system with a cost of approximate $\$ 10000$ US dolars is an cost-effective system. For data processing, we combine the mounting parameters and sensor parameters to $3 \mathrm{D}$ point cloud generating process. All offset value between reference frame were measured. The generated point cloud is corrected by applying bias correction and 7 parameters transformation. The test area is not flat with less object. The result is validated using three different experimental setups with three various flight height which indicate that the most accurate and reliable results are obtained. The point clouds after correcting attained an accuracy of approximate $0.2 \mathrm{~m}$ in vertical and horizontal.

In this study, the bias correction method is good to achieve the accuracy of less than $0.3 \mathrm{~m}$. The point cloud accuracy is not good enough for generating the topographic map at large scale. However, the stable results and the present accuracy are good for monitoring the crop growth. The obtained LiDARbased 3D point cloud can be combined with information from other sensors, such as RGB cameras and hyperspectral sensors which are also mounted all this UAV, to extract more valuable information related to different applications. In near future, we expect to use this system for monitoring the rice growth in Niigata prefecture, Japan.

\section{Acknowledgement}

This study was supported by Ho chi Minh City University of Technology-VNU-HCM under grant number T-KTXD-2018-113 and JSPS KAKENHI Grant Number 18K05901. Our sincere thanks also 
go to Mr. Nakamura and Mr. Yamane helping us carry out the field observation. Without their support, it would not be possible to conduct this research.

\section{Reference}

Adão, T., Hruška, J., Pádua, L., Bessa, J., Peres, E., Morais, R. and Sousa, J., 2017, Hyperspectral Imaging: a Review on UAV-Based Sensors, Data Processing and Applications for Agriculture and Forestry. Remote Sensing, 2017. 1110. 10.3390/rs9111110.

Angel, Y., Turner, D., Parkes, S., Malbeteau, Y., Lucieer, A. and McCabe, M., 2019, Automated Georectification and Mosaicking of UAV-Based Hyperspectral Imagery from Push-Broom Sensors. Remote Sensing, Vol. 12, 34. 10.3390/rs12010034.

Ehlert, D., Heisig, M. and Adamek, R., 2010, Suitability of a Laser Rangefinder to Characterize Winter Wheat. Precision Agriculture, Vol. 11, 650-663.

Glennie, C., Kusari, A. and Facchin, A., 2016, Calibration and Stability Analysis of the vlp-16 Laser Scanner. ISPRS - International Archives of the Photogrammetry, Remote Sensing and Spatial Information Sciences. XL-3/W4. 55-60. 10.5194/isprsarchives-XL-3-W4-55-2016.

Hosoi, F. and Omasa, K., 2006, Voxel-based 3-D Modeling of Individual Trees for Estimating Leaf Area Density Using High-Resolution Portable Scanning Lidar, IEEE Transactions on Geoscience and Remote Sensing, Vol. 44(12), 3610-3618.

Hosoi, F. and Omasa, K., 2009, Estimating Vertical Plant Area Density Profile and Growth Parameters of a Wheat Canopy at Different Growth Stages Using Three-Dimensional Portable Lidar Imaging. ISPRS Journal of Photogrammetry and Remote Sensing, Vol. 64, 151-158.

Ma, R., 2005, DEM Generation and Building Detection from Lidar Data. Photogrammetric Engineering \& Remote Sensing, Vol. 71(7), 847854.

Maan, G. S., Signh, C. K., Singh, M. K. and Nagarajan, B., 2015, Tree Species Biomass and Carbon Stock Measurement Using Ground Based-LiDAR. Geocarto International, Vol. 30(3).
Mcintosh, K., Krupnik, A. and Schenk, A, 2000, Improvement of Automatic DSM Generation Over Urban Areas Using airborne Laser Scanner Data. International Archives of Photogrammetry and Remote Sensing, Vol. XXXIII, Part B3, 563570.

Niethammer, U., James, M. , Rothmund, S., Travelletti, J. and Joswig, M., 2012, UAV-based Remote Sensing of the Super-Sauze Landslide: Evaluation and Results. Engineering Geology, Vol. 128, 2-11. 10.1016/j.enggeo.2011.03.012.

Ravi, R., Shamseldin, T., Elbahnasawy, M., Lin, Y. J. and Habib, A., 2018, Bias Impact Analysis and Calibration of UAV-Based Mobile LiDAR System with Spinning Multi-Beam Laser Scanner. Applied Sciences, Vol. 8. 297. 10.3390/app8020297.

Rice, S. K., Gutman, C. and Krouglicof, N., 2005, Laser Scanning Reveals Bryophyte Canopy Structure. The New Phytologist. Vol. 166(2), 695-704. http://www.jstor.org/stable/1514711.

Sibona, E., Vitali, A., Meloni, F., Caffo, L., Dotta, A., Lingua, E., Motta, R. and Garbarino, M., 2017, Direct Measurement of Tree Height Provides Different Results on the Assessment of LiDAR Accuracy. Forests, Vol. 8(7), doi:10.3390/f8010007.

Velodyne LiDAR, Inc, 2018., Velodyne manual, https://velodynelidar.com/downloads/, accessed on Dec., 2019.

Zawawi, A. A., Shiba, M. and Jemali, N, 2015, Accuracy of LiDAR-based Tree Height Estimation and crown Recognition in a Subtropical Evergreen Broad-Leaved Forest in Okinawa, Japan. Forest Systems, Vol. 24(1), http://dx.doi.org/10.5424/fs/2015241-05476. 